\title{
THE RESPONSIBILITY OF THE STUDENTS WITH MEDICAL SPECIALTIES FOR THEIR OWN HEALTH - REALITY AND OPPORTUNITIES FOR PREVENTION
}

\author{
S. Kyuchukova* \\ Department Health care, Medicine Facultacity, Trakia University, Stara Zagora, Bulgaria
}

\begin{abstract}
Striving to protect the health of young people is care not only of the individual but also of the state as an institution, and it has its duty and policy.

At the stage of studying at a Higher Medical School, the health of students is subject to the influence of various factors from their formal and informal living environment that would not be available if they did not study that. The main features are: working in hospital environment, continuous contact with patients who are healthy or ill, contact with biological materials, emergency situations, etc. This poses high responsibility for students with medical specialties and the implementation of special measures for the protection and care of their own health. That is why we set ourselves the following goal: To examine different aspects from the health of students with medical specialties related to individual behavioral factors and personal responsibility for their health.

Methods: Questionnaire survey; mathematical and statistical processing of the results; desk-research.

They made the following conclusions for students with medical specialties:

- They have responsible attitude towards their own health;

- They use strictly personal protective equipment at work in hospital environment;

- They are well informed about the issues of healthy nutrition, working and resting, avoiding stress;
\end{abstract}

Key words: students with medical specialties; responsibility; health; behavioral factors;

Ancient doctors have found that a man in his existence and his environment, constantly interact. Hippocrates, called the "father" of medicine, claims that being healthy means achieving harmony with yourself and the surrounding world. He defines health as a dynamic equilibrium achieved through life in harmony with natural laws. According to him, what is happening in mind, affects the body and a man must be seen in unity with the environment where he lives and works.

Striving to protect the health of young people is care not only of the individual but also of the state as an institution, and it has its duty and policy. Legislative and regulatory acts - Law for Youth, National Health Strategy with a second main strategic goal, etc., outline the directions

\footnotetext{
*Correspondence to: Kyuchukova Silviya, Trakia University, Medicine Facultacity, Department Health care, Bulgaria, Stara Zagora, Armeyska 9 street, tel.: +359898636 514; e-mail:

silviya.kyuchukova@trakia-uni.bg
}

and highlights in the actions of the governments as a whole.

At the stage of studying at a Higher Medical School, the health of students is subject to the influence of various factors from their formal and informal living environment that would not be available if they did not study that. The main features are: working in hospital environment, continuous contact with patients who are healthy or ill, contact with biological materials, emergency situations, etc. This poses high responsibility for students with medical specialties and the implementation of special measures for the protection and care of their own health. That is why we set ourselves the following goal: To examine different aspects from the health of students with medical specialties related to individual behavioral factors and personal responsibility for their health. 


\section{METHODS}

Questionnaire survey; mathematical and statistical processing of the results - IBM SPSS version 20; desk-research.

\section{Respondents}

155 Students of Thracian University, Medical faculty / specialties: nurse, midwife, physiotherapist, medical assistant, clinical laboratory /, Stara Zagora, Bulgaria.

Time interval: School year 2017/2018

In order to fulfill the purpose and the objectives of the present study, a special, adapted questionnaire was developed to collect the necessary information. It contained 15 questions, conditionally divided into 3 categories.

1 Group of questions: Personal protective equipment:

- What protective equipment do you use at work in a hospital ward?

How often do you wash your medical workwear?

$\square \quad$ What would be your behavior if you get an injury while doing a manipulation?

$\square \quad$ Others

2 Group of questions: Nosocomial / IH infections

口 In working with patients, when do you wash your hands hygienically?

What specific behavior would you perform to avoid IHI / internal hospital infections?

$\square \quad$ Do you think that the low kept manicure would help to protect your own health?

\section{O Others}

3 Group of questions: Lifestyle

- How do you cope with the daily stress?

口 Do you think that keeping healthy nutritional daily regime impacts your own health and prosperity?

口 Would you like to be a subject of prophylactic examinations, related to certain illnesses during your studies?

\section{$\square \quad$ Others}

In the process of his long research, G. L. Apasinenko has repeatedly concluded and emphasized that nowadays in the educational system lacks the necessary and sufficient level of knowledge to preserve and strengthen health and to improve the quality of life. As a result, it is not possible to take proper management decisions in the directions for implementing a healthy lifestyle strategy (1). This author is of the first ones, who talks about the sanocentric approach in medicine and this approach is incorrectly identified with holistic medicine. According to him, the lack of practical scientific research based on sanocentric health strategy reflects in poor information policy and enlightenment of the population.

In Directive 89/391/EO /measures for encouraging and improving health and safety of the employees at work/ and in The EU Strategic Framework for Health and Safety Working Conditions /HSWC/ 2014-2020 is indicated that each employee is responsible as much as possible for his own health and safety and for his colleagues $(2,3)$.

Everyone is obliged to protect, strengthen and be responsible for their own health and the health of their family (4).

Striving to protect the health of young people is care not only of the individual but also of the state as an institution, and it has its duty and policy. Legislative and regulatory acts regulates this:

Youth Law (5);
National Health Strategy with Second
Basic Strategic Objective:
Encouraging the healthy lifestyle;
Prevention of the factors, creating risk
for health;

- $\quad$ Conducting campaigns and initiatives to develop knowledge, attitudes and skills for healthy lifestyle;

- $\quad$ Safe behavior and avoiding risky health practices, etc.(3);

口 National youth program (2016-2020) with strategic objective: Encouraging the healthy lifestyle among young people

- Operative objective: Prevention of the factors, creating risk for health of the young people;

- $\quad$ Conducting campaigns and initiatives to develop knowledge, attitudes and skills for healthy lifestyle, safe behavior and avoiding risky health practices;

- Giving the youth people opportunities for education of young people, related to health, sport and physical activity (6).

\section{- Others}

The "others" section also includes the Compulsory Health Insurance Law, where one of the principles of the mandatory health insurance is "the responsibility of the insured people for their own health". In general, this obligation includes a system of care and attitude, and behavior (actions and inactions) aimed at protecting personal health. Here also possible costs should be included - not just money, but also time that a person spends to be healthy. In today's civilized world, young people are given a choice: to take care of their own health consciously or to leave it in the others' hands. The personal responsible choice actively 
involves the student himself in a serious step towards his personal growth. Taking personal responsibility for health, based on goodwill and awareness, brings only positive dividends.

The challenges posed before the youth policy for healthy lifestyle were the basis of the questions from the third group in the questionnaire and, in general, they formed the following question: Do students with medical specialties have a critical interest and concern for their own health? Focusing on the regular lifestyle and healthy nutrition, we asked the respondents: Do you think that keeping a healthy nutrition during the day affects your personal health and prosperity? Our expectations were surpassed by the high percentage of answers in the "Yes" area - 84\%. The results are presented in Figure 1.

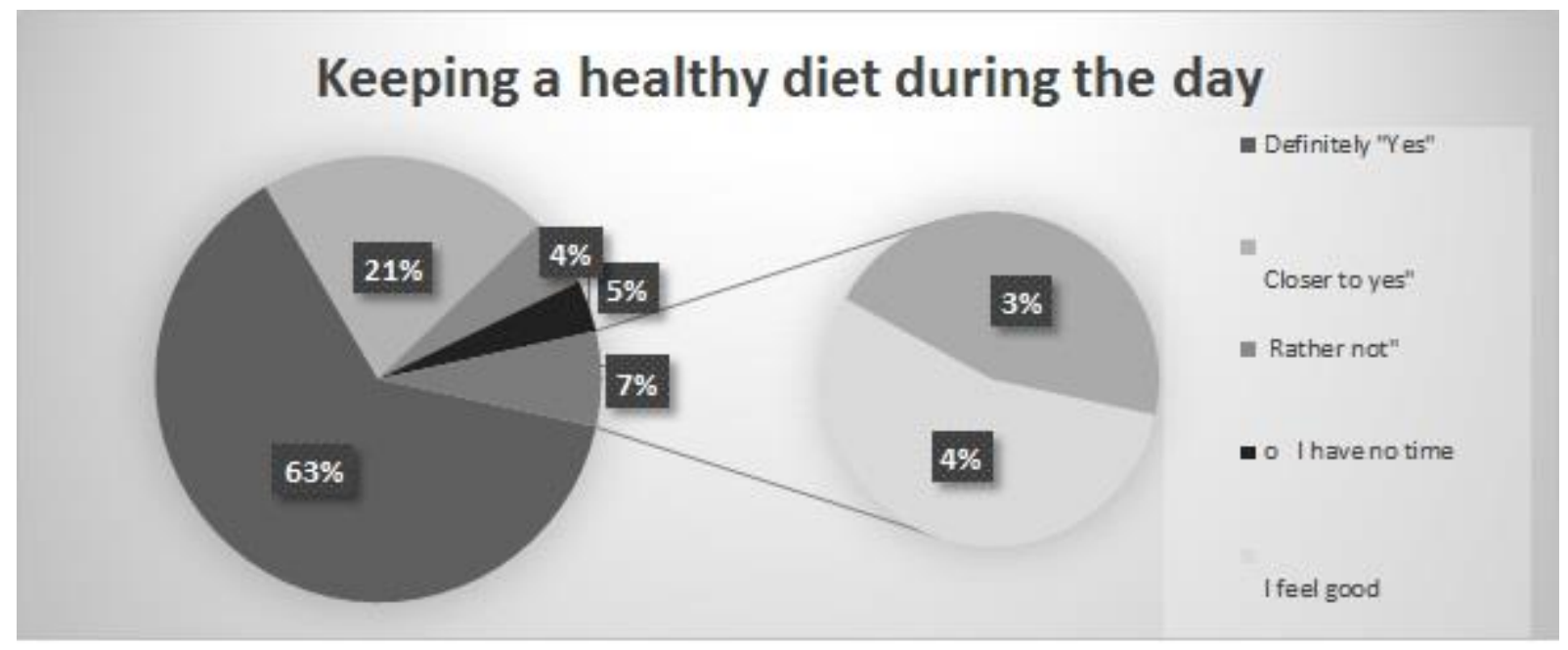

Figure 1. Keeping a healthy diet during the day

Those who do not keep a certain nutritional regime are $16 \%$. Of these, the possible reasons are divided into the $3-5 \%$ range shown on the graph. In a study in 2014, A. Andonova found out that students from the same and related disciplines had relatively high level of awareness of beneficial and harmful food, they actually assessed their nutrition with its positive and negative aspects, as approximately $75 \%$ of them partially respected the principles of healthy nutrition (7). As the time is passing by, in the case - for 4 years, the percentage of the respondents in the appropriate nutrition group has increased with ten more.

For most questions could be gathered information from the direct observations on behavior and actions of the students during their work in the training clinical bases. About question 5: How often do you wash your medical workwear?, we relied only on the correct answers of the respondents See Figure 2.

\section{Frequency of washing of working clothes}

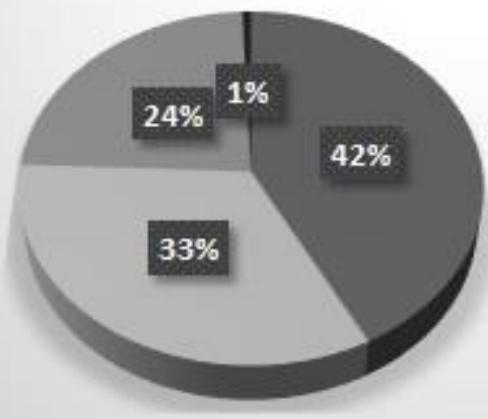

Immediately atter the work

an 2 times a week

at the end of theweek

- It is not necessary

Figure 2. Frequency of washing of working clothes 
The diagram shows that less than half $-42 \%$ of the respondents are doing exactly what we think is correct about the washing regime of workwear. One third of the respondents - 33\% wash their workwear twice a week, which we can accept in the norm of good practice, considering the fact that they attend clinical practice average twice a week. Only a quarter of respondents choose to do laundry washing at the end of the week. Those who have chosen the answer "not necessary" are only $1 \%$ of all respondents. I.e. two-thirds of the students, participated in the survey hygienically treat their workwear, according to the accepted standards and good practice for disinfection and sterilization of the workwear. In synchronization with the sanocentric approach in medicine and focusing on health promotion, the conditional third group of questions focused on the healthy lifestyle of the respondents. Taking into account the fact that during their training the students study disciplines, such as: Health Promotion, Healing and Dietetic Nutrition, Social Medicine and other subjects related to positive health and the prophylactics of a healthy lifestyle, we asked them: In your opinion, is it necessary more detailed training, concerning events aimed at improving the quality of life and protecting the personal health among students with medical specialties? The answers are presented in Figure 3.

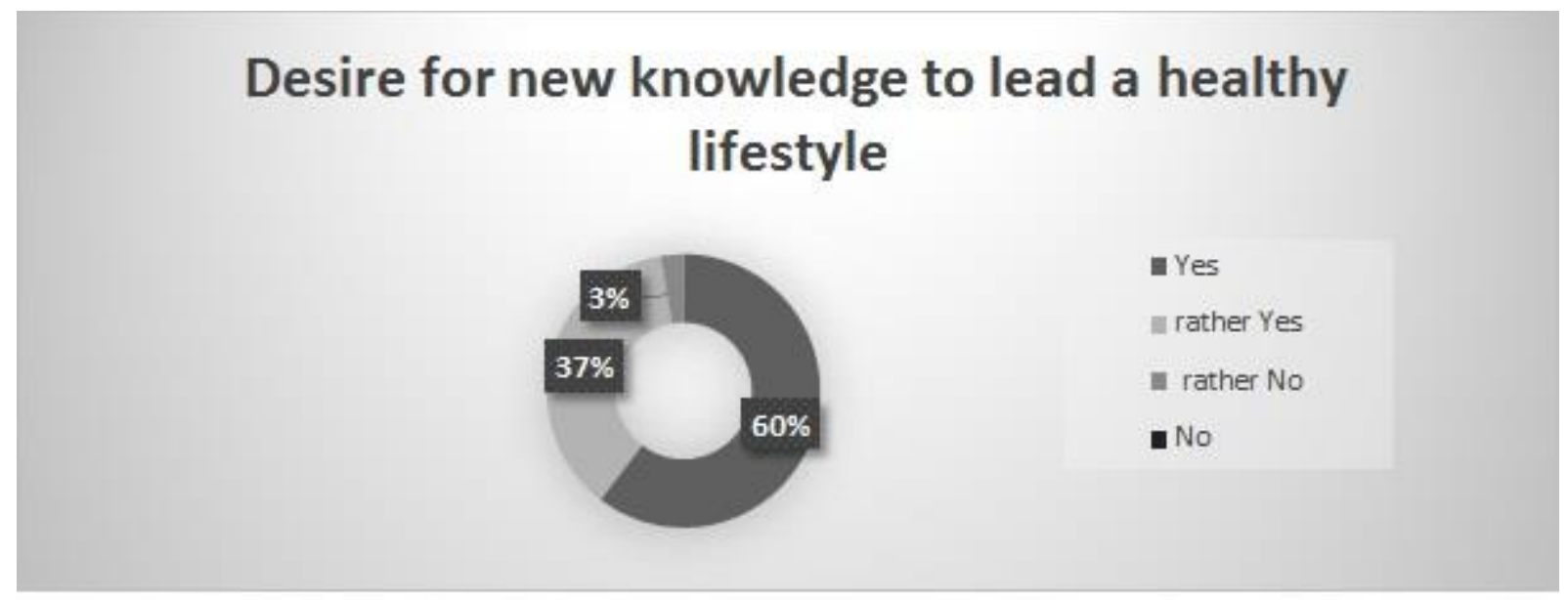

Figure 3. Desire for new knowledge to lead a healthy lifestyle

Here the answers with a tendency towards "Yes" stand out clearly, totally - 97\%, of them - $60 \%$ definitely "yes" and 37\% "rather yes". This extremely high percentage of positive students to acquire new and practically relevant knowledge about the healthy lifestyle is satisfying. Only 3\% of respondents are unwilling to supplement their awareness of these topics.

These answers directly correspond to the last question in the questionnaire asking participants to accept or reject the statement that "prophylaxis is better than cure". The results obtained are analogous to the previous ones, the difference is $1 \%$. The categorical supporters of this statement are $93 \%$ and those who are in contrary, are $7 \%$.

From the answers to the previous two questions, we can certainly assert that the students with medical specialties are convinced of the benefits of preventive medicine and prophylaxis, which are aimed at improving and preserving health personal health and that of the surrounding ones. At the same time, they want to be further trained on topics related to protecting of their own health.

After the provided research on the subject for the responsibility of the students with medical specialties for their own health and the results obtained, the following conclusions were made:

They have a responsible attitude towards their own health;

They strictly use personal protective equipment at work in a hospital environment;

$\square$ They are well informed about the issues of healthy eating, working and resting regime, stress avoidance;

$\square$ They want to be further trained on topics related to protecting of their own health. 


\section{CONCLUSION}

Students with medical specialties have learned and realized that health is not a constant magnitude. They take it as a dynamic equilibrium, which, in order to maintain, requires constant and conscious efforts. At the same time, they are clearly aware of the fact that the lack of disease and the affluent lifestyle are not a measure of good health.

The physically healthy person, who is devastating in his actions towards himself and others, is deeply ill in his nature. That is why, the responsibility for their own health, HMU students take with care and professionalism. For them, health is not only the absence of illness or disability, but is an expression of the way of thinking and the content of life.

\section{REFERENCES}

1. Apanasenko, G. L. , Sanocentric health strategy: a direct path to health of the population, Medico Informatics and Energetics, №3, DOI: http://dx.doi.org/10.11603/mie.19961960.2016.3.6750, 2016

2. http://publications.europa.eu/resource/celex/3 1989L039

3. http://ec.europa.eu/social/main.jsp?catId=151 \&langId=en

4. Apanasenko, G. L., Epidemiya khronicheskikh neinfektsionnykh zabolevanii: strategiya vyzhivaniya [The
KYUCHUKOVA S.

epidemic of chronic non-communicable diseases: a survival strategy]. Saarbrukken: Lambert Academic Publishing [in Russian], 2014

5. State Gazette 31/20.04.2012

6. http://mpes.government.bg/Documents/Progr ams/2016/PM(16_20)/NPM_2016-2020.pd

7. Andonova, A. Information and awareness of the students'healthy eating. Trakia Journal of Sciences, 12.suppl 1: 362-366, 2014

8. Andonova, A., S. Kuychukova and colleagues, Health knowledge for small and large, Training aid, Publishing house. IK KOTA, Stara Zagora, ISBN 978-954-305444-2, p. 64, 2014

9. Bauer, E.,Teoreticheskaya biologiya [Theoretical biology], Leningrad: AIEM [In Russian], 1935

10.Ivanova P., S. Kyuchukova, Risk of food disorders in healthcare students, 40th Science and Technology Session CONTACT 2017, Publishing. TEMTO, Sofia,pp. 100-105, ISSN 1313-9134, 2017

11.Spear B. B. Clinical application of pharmacogenetics / B. B. Spear, M. HeathChiozzi, J. Huff., Trends Mol. Med., Vol. 7, No. 5. - P. 201-204. doi: 10.1016/S14714914(01)01986-4, 2001

12.http://www.ncphp.government.bg/files/nczi/ Health2020_BG(1).pdhttp://www.puls.bg/hea lth/hot-news/news_18682.html 